\title{
OPTIMAL PREMIUM PLANS FOR REINSURANCE WITH REINSTATEMENTS
}

\author{
BY
}

\author{
Klaus Th. Hess and Klaus D. Schmidt
}

\begin{abstract}
The present paper is concerned with optimal premium plans for a reinsurance contract with reinstatements. In the reinsurance contract considered here, constant reinstatement premiums are due when the reinsurer's loss exceeds certain bounds. For this reinsurance contract we examine the existence of a premium plan which minimizes the expected squared difference between the loss and the total premium income of the reinsurer. We show that an optimal premium plan exists, that it is unique, and that it satisfies the net premium principle.
\end{abstract}

\section{INTRODUCTION}

The present paper is concerned with optimal premium plans for a reinsurance contract with reinstatements.

In the reinsurance contract considered here, constant reinstatement premiums are due when the reinsurer's loss exceeds certain bounds. For this reinsurance contract we examine the existence of a premium plan which minimizes the expected squared difference between the loss and the total premium income of the reinsurer. We show that an optimal premium plan exists, that it is unique, and that it satisfies the net premium principle. We also show that the optimal premium plan can be calculated from the first and second moments of certain functions of the reinsurer's loss.

It should be pointed out that, due to the use of constant reinstatement premiums, the reinsurance contract considered in this paper is simpler and more transparent than the familiar contracts in which the reinstatement premiums are calculated pro rata capita or pro rata temporis. In the first case, the reinstatement premiums depend on the size of a claim already occurred; in the second case, they depend on the time left after the occurrence of this claim. This means that in both cases the reinstatement premiums are random because of the randomness of the size and the occurrence time of a claim; they are thus unknown at the beginning of the contract.

This paper is organized as follows: In Section 2 we define the reinsurance contract and the optimization problem. In Section 3 we show that the optimization problem has a unique solution and that the solution satisfies the net 
premium principle and can be calculated from the first and second moments of certain functions of the reinsurer's loss; these results are obtained by solving an abstract credibility problem. In Section 4 we examine the properties of the optimal premium plan; the main result of this section asserts that premiums of the optimal premium plan are indeed nonnegative, although this condition is not part of the optimization problem. In Section 5 we present an alternative proof of our results which relies on the theory of conditional expectations. In Section 6 we give an example which illustrates the general results. We conclude with some remarks in Section 7.

Throughout this paper, let $(\Omega, \mathcal{F}, P)$ be a probability space.

\section{A Reinsurance Contract with Reinstatements}

We consider a real number $H \in(0, \infty)$ and a random variable $S: \Omega \rightarrow \mathbf{R}$ with $P[\{0 \leq S \leq H\}]=1$. The random variable $S$ is interpreted as the reinsurer's aggregate loss and the real number $H$ is interpreted as the aggregate limit of the reinsurer's liability.

2.1. Example (Excess-of-Loss Reinsurance). Assume that the aggregate loss $X^{\prime}$ of the cedent can be represented as

$$
X^{\prime}=\sum_{j=1}^{N^{\prime}} Y_{j}^{\prime}
$$

where

- $N^{\prime}$ is the (random) number of claims and

- $Y_{j}^{\prime}$ is the (random) size of the $j$-th claim

such that the sequence $\left\{Y_{j}^{\prime}\right\}_{j \in \mathbf{N}}$ is i.i.d. and independent of $N^{\prime}$. This means that the pair $\left\langle N^{\prime},\left\{Y_{j}^{\prime}\right\}_{j \in \mathrm{N}}\right\rangle$ is a collective model for the cedent's loss $X^{\prime}$. In an excess-of-loss reinsurance contract with priority $d \in(0, \infty)$ and limit $h \in(0, \infty)$, the reinsurer's aggregate loss is given by

$$
X:=\sum_{j=1}^{N^{\prime}} \min \left\{\left(Y_{j}^{\prime}-d\right)^{+}, h\right\}
$$

The random variables of the collective model for the cedent's loss cannot be observed by the reinsurer, but it has been shown by Hess [2003] that the collective model $\left\langle N^{\prime},\left\{Y_{j}^{\prime}\right\}_{j \in \mathbf{N}}\right\rangle$ can be transformed into a collective model $\left\langle N,\left\{Y_{j}\right\}_{j \in \mathbf{N}}\right\rangle$ where

$-N$ is the number of claims exceeding the priority and

$-Y_{j}$ is the size of the $j$-th claim exceeding the priority such that the reinsurer's loss can be represented as

$$
X=\sum_{j=1}^{N} \min \left\{Y_{j}-d, h\right\}
$$


(see also Schmidt [1996; Chapter 6] and Schmidt [2002; Kapitel 8]). If the reinsurance contract is also subject to an aggregate deductible $D \in[0, \infty)$ and an aggregate limit $H \in(0, \infty)$, then the reinsurer's loss is given by

$$
S:=\min \left\{(X-D)^{+}, H\right\}
$$

and the random variable $S$ satisfies $P[\{0 \leq S \leq H\}]=1$.

We consider a reinsurance contract on $S$ with $n \in \mathbf{N}_{0}$ reinstatements in which

- the global layer $[0, H]$ is partitioned into the $n+1$ layers $[0, h],(h, 2 h], \ldots$, $(n h, H]$ with

$$
h:=\frac{H}{n+1}
$$

- an initial premium $\pi_{0} \in \mathbf{R}$ is due at the beginning of the contract, and

- a reinstatement premium $\pi_{k} \in \mathbf{R}$ is due when the loss $S$ exceeds the value $k h$ with $k \in\{1, \ldots, n\}$.

Every finite sequence $\pi=\left\{\pi_{k}\right\}_{k \in\{0,1, \ldots, n\}} \subseteq \mathbf{R}$ is said to be a premium plan for a reinsurance contract with $n$ reinstatements.

For $k \in\{0,1, \ldots, n\}$, define

$$
\alpha_{k}:= \begin{cases}P[\{0 \leq S \leq h\}] & \text { if } k=0 \\ P[\{k h<S \leq(k+1) h\}] & \text { if } k \in\{1, \ldots, n\}\end{cases}
$$

Then we have $\sum_{k=0}^{n} \alpha_{k}=1$.

We assume henceforth that $\alpha_{k} \in(0,1)$ holds for all $k \in\{0,1, \ldots, n\}$.

2.2. Example (Excess-of-Loss Reinsurance). In the case $D=0$ the reinsurer's loss can be written as

$$
S=\sum_{m=0}^{\infty} \chi_{\{N=m\}} \min \left\{\sum_{j=1}^{m} \min \left\{Y_{j}-d, h\right\}, H\right\}
$$

(where $\chi_{\{N=m\}}$ is the random variable which is equal to 1 if $N=m$ and equal to 0 if $N \neq m$ ). If at least $m$ claims exceeding the priority occur and if the $m$-th claim exceeding the priority is such that $\sum_{j=1}^{m-1} \min \left\{Y_{j}-d, h\right\} \leq k h<\sum_{j=1}^{m} \min$ $\left\{Y_{j}-d, h\right\}$, then the reinstatement premium $\pi_{k}$ is due.

Let $\Pi$ denote the collection of all premium plans for a reinsurance contract with $n$ reinstatements. For a premium plan $\pi=\left\{\pi_{k}\right\}_{k \in\{0,1, \ldots, n\}} \in \Pi$, the total premium of $\pi$ is defined as the random variable

$$
\delta(\pi):=\pi_{0}+\sum_{k=1}^{n} \pi_{k} \chi_{\{k h<S\}}
$$


and the expected squared prediction error of $\pi$ is defined as

$$
E\left[(\delta(\pi)-S)^{2}\right]
$$

A premium plan $\pi=\left\{\pi_{k}\right\}_{k \in\{0,1, \ldots, n\}} \in \Pi$ is said to be

- unbiased if

$$
E[\delta(\pi)]=E[S]
$$

(which means that $\pi$ satisfies the net premium principle),

- nonnegative if

$$
\pi_{k} \geq 0
$$

holds for all $k \in\{0,1, \ldots, \mathrm{n}\}$,

- optimal if it minimizes the expected squared prediction error over all premium plans.

We shall show that

- there exists a unique premium plan $\pi^{*}=\left\{\pi_{k}^{*}\right\}_{k \in\{0,1, \ldots, n\}} \in \Pi$ which minimizes the expected squared prediction error over all $\pi \in \Pi$,

- the premium plan $\pi^{*}$ is unbiased, and

- the premium plan $\pi^{*}$ is nonnegative.

\section{Existence and Uniqueness of an Optimal Premium Plan}

For every premium plan $\pi=\left\{\pi_{k}\right\}_{k \in\{0,1, \ldots, n\}}$, the total premium

$$
\delta(\pi)=\pi_{0}+\sum_{k=1}^{n} \pi_{k} \chi_{\{k h<S\}}
$$

is an affine-linear function of the explanatory random variables $\left.\chi_{\{h<S\}}, \ldots, \chi_{\{h n}<S\right\}$. Therefore, the problem of minimizing the expected squared prediction error

$$
E\left[(\delta(\pi)-S)^{2}\right]
$$

over all premium plans $\pi \in \Pi$ can be viewed as a credibility problem.

It is well-known that the credibility problem always has a unique solution. Moreover, if the variance of the random vector formed by the explanatory variables is invertible, then the solution has a unique representation as an affine-linear function of the explanatory random variables, and if the inverse of the variance is known, then an explicit formula can be given for the coefficients of the solution.

Define

$$
\mathbf{X}:=\left(\begin{array}{c}
\chi_{\{h<s\}} \\
\vdots \\
\chi_{\{n h<s\}}
\end{array}\right)
$$


as well as

$$
\begin{aligned}
\boldsymbol{\mu} & :=E[\mathbf{X}] \\
\mu & :=E[S]
\end{aligned}
$$

and

$$
\begin{aligned}
\boldsymbol{\Sigma} & :=\operatorname{Var}[\mathbf{X}] \\
\boldsymbol{\rho} & :=\operatorname{Cov}[\mathbf{X}, S] \\
\sigma^{2} & :=\operatorname{var}[S]
\end{aligned}
$$

Our aim is to show that $\boldsymbol{\Sigma}$ is invertible and to compute the inverse of $\boldsymbol{\Sigma}$.

For $k \in\{1, \ldots, n\}$, define a matrix $\mathbf{B}_{k} \in \mathbf{R}^{n \times n}$ by letting

$$
b_{k: i, j}:= \begin{cases}1 & \text { if } i, j \leq k \\ 0 & \text { else }\end{cases}
$$

and put

$$
\mathbf{A}:=\sum_{k=1}^{n} \alpha_{k} \boldsymbol{B}_{\boldsymbol{k}}
$$

We also define a matrix $\mathbf{G} \in \mathbf{R}^{n \times n}$ by letting

$$
g_{i, j}:= \begin{cases}\sum_{k=i}^{n} \alpha_{k} & \text { if } j=1 \\ 0 & \text { else }\end{cases}
$$

The following results shows that $\boldsymbol{\Sigma}$ can be expressed in terms of $\mathbf{A}$ and $\mathbf{G}$ :

3.1. Lemma. The matrix $\mathbf{\Sigma}$ satisfies

$$
\boldsymbol{\Sigma}=\mathbf{A}-\mathbf{G G}^{\prime}
$$

Proof. For all $i \in\{1, \ldots, n\}$ and $j \in\{1, \ldots, i\}$, we have

$$
X_{i} X_{j}=\chi_{\{i h<S\} \cap\{j h<S\}}=\chi_{\{i h<S\}}=X_{i}
$$

and hence

$$
E\left[X_{i} X_{j}\right]=E\left[X_{i}\right]=P[\{i h<S\}]=\sum_{k=i}^{n} \alpha_{k}
$$

This yields $E\left[\mathbf{X} \mathbf{X}^{\prime}\right]=\mathbf{A}$ and $E[\mathbf{X}](E[\mathbf{X}])^{\prime}=\mathbf{G G}^{\prime}$. The assertion follows. 
For $k \in\{0,1, \ldots, n\}$, define a matrix $\mathbf{C}_{k} \in \mathbf{R}^{n \times n}$ by letting

$$
c_{k: i, j}:=\left\{\begin{aligned}
1 & \text { if }(i, j) \in\{(k, k),(k+1, k+1)\} \\
-1 & \text { if }(i, j) \in\{(k, k+1),(k+1, k)\} \\
0 & \text { else }
\end{aligned}\right.
$$

Then we have $\mathbf{C}_{0}=\mathbf{B}_{1}$.

3.2. Lemma. The matrix $\mathbf{A}$ is invertible and its inverse satisfies

$$
\mathbf{A}^{-1}=\sum_{k=1}^{n} \alpha_{k}^{-1} \mathbf{C}_{k}
$$

Proof. For $k \in\{1, \ldots, n\}$, define a matrix $\mathbf{D}_{k} \in \mathbf{R}^{n \times n}$ by letting

$$
d_{k: i, j}:=\left\{\begin{aligned}
1 & \text { if } i \leq k=j \\
-1 & \text { if } i<k+1=j \\
0 & \text { else }
\end{aligned}\right.
$$

Then the identity

$$
\mathbf{B}_{k} \mathbf{C}_{l}= \begin{cases}\mathbf{D}_{k} & \text { if } k=l \\ \mathbf{O} & \text { else }\end{cases}
$$

holds for all $k, l \in\{1, \ldots, n\}$. We thus obtain

$$
\left(\sum_{k=1}^{n} \alpha_{k} \mathbf{B}_{k}\right)\left(\sum_{k=1}^{n} \alpha_{k}^{-1} \mathbf{C}_{k}\right)=\sum_{k=1}^{n} \mathbf{D}_{k}=\mathbf{I}
$$

as was to be shown.

3.3. Lemma. The matrix $\mathbf{\Sigma}$ is invertible and its inverse satisfies

$$
\boldsymbol{\Sigma}^{-1}=\sum_{k=0}^{n} \alpha_{k}^{-1} \mathbf{C}_{k}
$$

Proof. Since $\mathbf{G}=\mathbf{A B}_{1}$ and $\mathbf{B}_{1}$ is symmetric and idempotent, Lemma 3.1 yields

$$
\begin{aligned}
\boldsymbol{\Sigma} & =\mathbf{A}-\mathbf{G G}^{\prime} \\
& =\mathbf{A}-\left(\mathbf{A B}_{1}\right)\left(\mathbf{A} \mathbf{B}_{1}\right)^{\prime} \\
& =\mathbf{A}-\mathbf{A} \mathbf{B}_{1} \mathbf{A} \\
& =\mathbf{A}-\mathbf{G A} \\
& =(\mathbf{I}-\mathbf{G}) \mathbf{A}
\end{aligned}
$$


Using Lemma 3.2 and the identity $\mathbf{G}^{2}=\left(1-\alpha_{0}\right) \mathbf{G}$, we obtain

$$
\begin{aligned}
\boldsymbol{\Sigma}\left(\sum_{k=0}^{n} \alpha_{k}^{-1} \mathbf{C}_{k}\right) & =(\mathbf{I}-\mathbf{G}) \mathbf{A}\left(\alpha_{0}^{-1} \mathbf{B}_{1}+\mathbf{A}^{-1}\right) \\
& =(\mathbf{I}-\mathbf{G})\left(\alpha_{0}^{-1} \mathbf{G}+\mathbf{I}\right) \\
& =\alpha_{0}^{-1}\left(\mathbf{G}-\mathbf{G}^{2}\right)+(\mathbf{I}-\mathbf{G}) \\
& =\mathbf{G}+(\mathbf{I}-\mathbf{G}) \\
& =\mathbf{I}
\end{aligned}
$$

as was to be shown.

3.4. Theorem. There exists a unique premium plan $\pi^{*}=\left\{\pi_{k}^{*}\right\}_{k \in\{0,1, \ldots, n\}} \in \Pi$ which minimizes the expected squared prediction error and the total premium of the premium plan $\pi^{*}$ satisfies

$$
\delta\left(\pi^{*}\right)=\mu+\boldsymbol{\rho}^{\prime} \boldsymbol{\Sigma}^{-1}(\mathbf{X}-\boldsymbol{\mu})
$$

and

$$
E\left[\left(\delta\left(\pi^{*}\right)-S\right)^{2}\right]=\sigma^{2}-\boldsymbol{\rho}^{\prime} \Sigma^{-1} \boldsymbol{\rho}
$$

In particular, the initial premium $\pi_{0}^{*}$ satisfies

$$
\pi_{0}^{*}=\mu-\boldsymbol{\mu}^{\prime} \boldsymbol{\Sigma}^{-1} \boldsymbol{\rho}
$$

and the reinstatement premiums $\pi_{0}^{*}, \ldots, \pi_{n}^{*}$ satisfy

$$
\left(\begin{array}{c}
\pi_{1}^{*} \\
\vdots \\
\pi_{n}^{*}
\end{array}\right)=\boldsymbol{\Sigma}^{-1} \boldsymbol{\rho}
$$

Moreover, the premium plan $\pi^{*}$ is unbiased.

The proof of Theorem 3.4 is straightforward; see, for example, Hess and Schmidt [2001].

\section{Properties of the Optimal Premium Plan}

While Theorem 3.4 yields the existence and uniqueness of an optimal premium plan as well as an explicit formula for the initial premium and the reinstatement premiums of this premium plan, we still have to show that the optimal premium plan is nonnegative. This will be the main result of the present section. 
For $k \in\{0,1, \ldots, n, n+1\}$, define

$$
\rho_{k}:= \begin{cases}\operatorname{cov}\left[S, \chi_{\{k h<S\}}\right] & \text { if } k \in\{1, \ldots, n\} \\ 0 & \text { if } k \in\{0, n+1\}\end{cases}
$$

Then we have

$$
\boldsymbol{\rho}=\left(\begin{array}{c}
\rho_{1} \\
\vdots \\
\rho_{n}
\end{array}\right)
$$

The following result provides a formula for the optimal premium plan:

4.1. Theorem. The optimal premium plan $\pi^{*}=\left\{\pi_{k}^{*}\right\}_{k \in\{0,1, \ldots, n\}}$ satisfies

$$
\pi_{k}^{*}= \begin{cases}\mu-\frac{\rho_{1}}{\alpha_{0}} & \text { if } k=0 \\ \frac{\rho_{k}-\rho_{k+1}}{\alpha_{k}}-\frac{\rho_{k-1}-\rho_{k}}{\alpha_{k-1}} & \text { if } k \in\{1, \ldots, n\}\end{cases}
$$

Proof. For all $k \in\{0,1, \ldots, n\}$, we have

$$
\mathbf{C}_{k} \boldsymbol{\rho}=\left(\rho_{k}-\rho_{k+1}\right) \mathbf{c}_{k}-\left(\rho_{k}-\rho_{k+1}\right) \mathbf{c}_{k+1}
$$

with

$$
\mathbf{c}_{k}:= \begin{cases}\mathbf{e}_{k} & \text { if } k \in\{1, \ldots, n\} \\ 0 & \text { if } k \in\{0, n+1\}\end{cases}
$$

where $\mathbf{e}_{k}$ denotes the $k$-th unit vector of $\mathbf{R}^{n}$. Define

$$
\boldsymbol{\pi}^{*}=\left(\begin{array}{c}
\pi_{1}^{*} \\
\vdots \\
\pi_{n}^{*}
\end{array}\right)
$$

Using Theorem 3.4 and Lemma 3.3, we obtain

$$
\begin{aligned}
\boldsymbol{\pi}^{*} & =\boldsymbol{\Sigma}^{-1} \boldsymbol{\rho} \\
& =\sum_{k=0}^{n} \alpha_{k}^{-1} \mathbf{C}_{k} \boldsymbol{\rho} \\
& =\sum_{k=0}^{n} \alpha_{k}^{-1}\left(\left(\rho_{k}-\rho_{k+1}\right) \mathbf{c}_{k}-\left(\rho_{k}-\rho_{k+1}\right) \mathbf{c}_{k+1}\right) \\
& =\sum_{k=1}^{n}\left(\frac{\rho_{k}-\rho_{k+1}}{\alpha_{k}}-\frac{\rho_{k-1}-\rho_{k}}{\alpha_{k-1}}\right) \mathbf{e}_{k}
\end{aligned}
$$


This proves the identity for $\pi_{k}^{*}$ with $k \in\{1, \ldots, n\}$. Furthermore, we have

$$
\boldsymbol{\mu}=\sum_{k=1}^{n}\left(\sum_{j=k}^{n} \alpha_{j}\right) \mathbf{e}_{k}
$$

Using Theorem 3.4 again, we obtain

$$
\begin{aligned}
\pi_{0}^{*} & =\mu-\boldsymbol{\mu}^{\prime} \boldsymbol{\Sigma}^{-1} \boldsymbol{\rho} \\
& =\mu-\boldsymbol{\mu}^{\prime} \boldsymbol{\pi}^{*} \\
& =\mu-\sum_{k=1}^{n}\left(\sum_{j=k}^{n} \alpha_{j}\right)\left(\frac{\rho_{k}-\rho_{k+1}}{\alpha_{k}}-\frac{\rho_{k-1}-\rho_{k}}{\alpha_{k-1}}\right) \\
& =\mu-\sum_{j=1}^{n} \alpha_{j} \sum_{k=1}^{j}\left(\frac{\rho_{k}-\rho_{k+1}}{\alpha_{k}}-\frac{\rho_{k-1}-\rho_{k}}{\alpha_{k-1}}\right) \\
& =\mu-\sum_{j=1}^{n} \alpha_{j}\left(\frac{\rho_{j}-\rho_{j+1}}{\alpha_{j}}+\frac{\rho_{1}}{\alpha_{0}}\right) \\
& =\mu-\left(\rho_{1}+\left(1-\alpha_{0}\right) \frac{\rho_{1}}{\alpha_{0}}\right) \\
& =\mu-\frac{\rho_{1}}{\alpha_{0}}
\end{aligned}
$$

This proves the identity for $\pi_{0}^{*}$.

The following results gives another representation of the premiums of the optimal premium plan and shows that the optimal premium plan is nonnegative:

4.2. Theorem. The optimal premium plan $\pi^{*}=\left\{\pi_{k}^{*}\right\}_{k \in\{0,1, \ldots, n\}}$ satisfies

$$
\pi_{k}^{*}= \begin{cases}E[S \mid\{0 \leq S \leq h\}] & \text { if } k=0 \\ E[S \mid\{k h<S \leq(k+1) h\}]-E[S \mid\{(k-1) h<S \leq k h\}] & \text { if } k \in\{1, \ldots, n\}\end{cases}
$$

In particular, the optimal premium plan is nonnegative.

Proof. For all $k \in\{1, \ldots, n\}$, we have

$$
\begin{aligned}
E[S \mid\{k h<S \leq(k+1) h\}] & =\frac{E\left[S \chi_{\{k h<S \leq(k+1) h\}}\right]}{P[\{k h<S \leq(k+1) h\}]} \\
& =\frac{1}{\alpha_{k}}\left(E\left[S \chi_{\{k h<S\}}\right]-E\left[S \chi_{\{(k+1) h<S\}}\right]\right) \\
& =\frac{1}{\alpha_{k}}\left(\left(\rho_{k}+\mu \sum_{j=k}^{n} \alpha_{j}\right)-\left(\rho_{k+1}+\mu \sum_{j=k+1}^{n} \alpha_{j}\right)\right) \\
& =\mu+\frac{\rho_{k}-\rho_{k+1}}{\alpha_{k}}
\end{aligned}
$$


and the same argument yields

$$
E[S \mid\{0 \leq S \leq h\}]=\mu-\frac{\rho_{1}}{\alpha_{0}}
$$

The identity for the premiums of the optimal premium plan now follows from Theorem 4.1. Moreover, we have

$$
\begin{aligned}
E[S \mid\{k h<S \leq(k+1) h\}] & \geq k h \\
& \geq E[S \mid\{(k-1) h<S \leq k h\}]
\end{aligned}
$$

for all $k \in\{1, \ldots, n\}$, and we also have

$$
E[S \mid\{0 \leq S \leq h\}] \geq 0
$$

This implies the optimal premium plan is nonnegative.

Let us finally compute the expected squared prediction error and the variance of the total premium of the optimal premium plan:

4.3. Theorem. The expected squared prediction error of the total premium of the optimal premium plan satisfies the identity

$$
E\left[\left(\delta\left(\pi^{*}\right)-S\right)^{2}\right]=\sigma^{2}-\sum_{k=0}^{n} \alpha_{k}^{-1}\left(\rho_{k}-\rho_{k+1}\right)^{2}
$$

and the variance of the total premium of the optimal premium plan satisfies

$$
\operatorname{Var}\left[\delta\left(\pi^{*}\right)\right]=\sum_{k=0}^{n} \alpha_{k}^{-1}\left(\rho_{k}-\rho_{k+1}\right)^{2}
$$

Proof. For each $k \in\{0,1, \ldots, n\}$, we have

$$
\mathbf{C}_{k}^{2}=2 \mathbf{C}_{k}
$$

and well as

$$
\mathbf{C}_{k} \boldsymbol{\rho}=\left(\rho_{k}-\rho_{k+1}\right) \mathbf{c}_{k}-\left(\rho_{k}-\rho_{k+1}\right) \mathbf{c}_{k+1}
$$

(as shown in the proof of Theorem 4.1), and hence

$$
\begin{aligned}
\boldsymbol{\rho}^{\prime} \mathbf{C}_{k} \boldsymbol{\rho} & =\frac{1}{2} \boldsymbol{\rho}^{\prime} \mathbf{C}_{k}^{2} \boldsymbol{\rho} \\
& =\frac{1}{2}\left(\mathbf{C}_{k} \boldsymbol{\rho}\right)^{\prime}\left(\mathbf{C}_{k} \boldsymbol{\rho}\right) \\
& =\left(\rho_{k}-\rho_{k+1}\right)^{2}
\end{aligned}
$$


From Theorem 3.4 and Lemma 3.3 we now obtain

$$
\begin{aligned}
E\left[\left(\delta\left(\pi^{*}\right)-S\right)^{2}\right] & =\sigma^{2}-\boldsymbol{\rho}^{\prime} \boldsymbol{\Sigma}^{-1} \boldsymbol{\rho} \\
& =\sigma^{2}-\boldsymbol{\rho}^{\prime}\left(\sum_{k=0}^{n} \alpha_{k}^{-1} \mathbf{C}_{k}\right) \boldsymbol{\rho} \\
& =\sigma^{2}-\sum_{k=0}^{n} \alpha_{k}^{-1}\left(\rho_{k}-\rho_{k+1}\right)^{2}
\end{aligned}
$$

This proves the first identity and the second follows from the first.

\section{An Alternative Proof}

For readers who are familiar with the theory of conditional expectations with respect to a sub- $\sigma$-algebra $\mathcal{G}$ of $\mathcal{F}$, we present an alternative proof of the main results of this paper.

Our starting point is that, for every premium plan $\pi=\left\{\pi_{k}\right\}_{k \in\{0,1, \ldots, n\}} \in \Pi$, the total premium

$$
\delta(\pi)=\pi_{0}+\sum_{k=1}^{n} \pi_{k} \chi_{\{k h<S\}}
$$

can be written as

$$
\delta(\pi)=\pi_{0} \chi_{\{0 \leq S \leq h\}}+\sum_{j=1}^{n}\left(\sum_{k=0}^{j} \pi_{k}\right) \chi_{\{j h<S \leq(j+1) h\}}
$$

Since $\alpha_{0}, \alpha_{1}, \ldots, \alpha_{n} \in(0,1)$, we may identify the collection $\Pi$ of all premium plans with the set

$$
\Delta:=\operatorname{span}\left\{\chi_{\{0 \leq S \leq h\}}, \chi_{\{h<S \leq 2 h\}}, \ldots, \chi_{\{n h<S \leq(n+1) h\}}\right\}
$$

which is a closed linear subspace of the Hilbert space $L^{2}(\mathcal{F})$. The projection theorem in Hilbert spaces, applied to the reinsurer's loss $S$ and the closed linear subspace $\Delta$ of $L^{2}(\mathcal{F})$, yields the existence of a unique premium plan $\pi^{*} \in \Pi$ such that the total premium $\delta\left(\pi^{*}\right)$ minimizes the norm

$$
\|\delta(\pi)-S\|_{2}=\left(E\left[(\delta(\pi)-S)^{2}\right]\right)^{1 / 2}
$$

over all $\pi \in \Pi$.

Let $\mathcal{G}$ denote the sub- $\sigma$-algebra of $\mathcal{F}$ which is generated by the random variables $\left.\chi_{\{0 \leq S \leq h\}}, \chi_{\{h<S \leq 2 h\}}, \ldots, \chi_{\{n h<}<S \leq(n+1) h\right\}$. The conditional expectation $E^{\mathcal{G}}(S)$ of $S$ with respect to $\mathcal{G}$ minimizes the norm 


$$
\|Y-S\|_{2}=\left(E\left[(Y-S)^{2}\right]\right)^{1 / 2}
$$

over all $Y \in L^{2}(\mathcal{G})$. The random variables $\chi_{\{0 \leq S \leq h\}}, \chi_{\{h<S \leq 2 h\}}, \ldots, \chi_{\{n h<S \leq(n+1) h\}}$ are nonzero and orthogonal in $L^{2}(\mathcal{F})$. Therefore, we have $L^{2}(\mathcal{G})=\Delta$ and the conditional expectation of $S$ with respect to $\mathcal{G}$ can be written as

$$
\begin{aligned}
E^{\mathcal{G}}(S) & =E[S \mid\{0 \leq S \leq h\}] \chi_{\{0 \leq S \leq h\}} \\
& +\sum_{j=1}^{n} E[S \mid\{j h<S \leq(j+1) h\}] \chi_{\{j h<S \leq(j+1) h\}}
\end{aligned}
$$

In particular, we have $E^{\mathcal{G}}(S) \in \Delta$ and hence

$$
E^{\mathcal{G}}(S)=\delta\left(\pi^{*}\right)
$$

Since $\left.E\left[\delta\left(\pi^{*}\right)\right]=E\left[E^{\mathcal{G}}(S)\right)\right]=E[S]$, the optimal premium plan is unbiased. Since the conditional expectation $E^{\mathcal{G}}: L^{2}(\mathcal{F}) \rightarrow L^{2}(\mathcal{G})$ is a positive operator, the optimal premium plan $\pi^{*}$ is nonnegative.

\section{An ExAmple}

To illustrate the general results, we consider the case where the reinsurer's loss has a truncated exponential distribution:

6.1. Example (Truncated Exponential Distribution). Consider a random variable $X$ with

$$
P[\{X \leq x\}]= \begin{cases}0 & \text { if } x \leq 0 \\ \int_{0}^{x} \alpha e^{-\alpha t} d t & \text { if } x>0\end{cases}
$$

for some parameter $\alpha \in(0, \infty)$, which means that the distribution of $X$ is the exponential distribution with parameter $\alpha$. Define

$$
S:=\min \{X, H\}
$$

Then the net premium for $S$ is

$$
E[S]=\frac{1}{\alpha}\left(1-e^{-\alpha H}\right)
$$

With $k \in\{1, \ldots, n-1\}$, we have

$$
\begin{aligned}
P[\{0 \leq S \leq h\}] & =1-e^{-\alpha h} \\
P[\{k h<S \leq(k+1) h\}] & =e^{-\alpha k h}-e^{-\alpha(k+1) h} \\
P[\{n h<S \leq H\}] & =e^{-\alpha n h}
\end{aligned}
$$


and

$$
\begin{aligned}
E\left[S \chi_{\{0 \leq S \leq h\}}\right] & =\frac{1}{\alpha}\left(1-e^{-\alpha h}\right)-h e^{-\alpha h} \\
E\left[S \chi_{\{k h<S \leq(k+1) h\}}\right] & =\frac{1}{\alpha}\left(e^{-\alpha k h}-e^{-\alpha(k+1) h}\right)+\left(k h e^{-\alpha k h}-(k+1) h e^{-\alpha(k+1) h}\right) \\
E\left[S \chi_{\{n h<S \leq H\}}\right] & =\frac{1}{\alpha}\left(e^{-\alpha n h}-e^{-\alpha H}\right)+n h e^{-\alpha n h}
\end{aligned}
$$

and hence

$$
\begin{aligned}
E[S \mid\{0 \leq S \leq h\}] & =\frac{1}{\alpha}-\frac{h e^{-\alpha h}}{1-e^{-\alpha h}} \\
E[S \mid\{k h<S \leq(k+1) h\}] & =\frac{1}{\alpha}-\frac{h e^{-\alpha h}}{1-e^{-\alpha h}}+k h \\
E[S \mid\{n h<S \leq H\}] & =\frac{1}{\alpha}-\frac{e^{-\alpha h}}{\alpha}+n h
\end{aligned}
$$

Therefore, the premiums of the optimal premium plan $\pi^{*}=\left\{\pi_{k}^{*}\right\}_{k \in\{0,1, \ldots, n\}}$ satisfy

$$
\pi_{k}^{*}= \begin{cases}\frac{1}{\alpha}-\frac{h e^{-\alpha h}}{1-e^{-\alpha h}} & \text { if } k=0 \\ h & \text { if } k \in\{1, \ldots, n-1\} \\ \frac{h}{1-e^{-\alpha h}}-\frac{e^{-\alpha h}}{\alpha} & \text { if } k=n\end{cases}
$$

and hence $\pi_{0}^{*}<h<\pi_{n}^{*}$.

Explicit formulas for the premiums of the optimal premium plan can also be obtained in the case where the reinsurer's loss has a truncated Pareto distribution.

\section{REMARKS}

Reinsurance contracts with different numbers of reinstatements are difficult to compare. However, every premium plan for a reinsurance contract with no reinstatements $(n=0)$ can be extended to a premium plan for a reinsurance contract with one (free) reinstatement $(n=1)$. Therefore, the optimal premium plan for a reinsurance contract with one reinstatement is at least as good as the optimal premium plan for a reinsurance contract with no reinstatements. More generally, the optimal premium plan for a reinsurance contract with $2 n+1$ reinstatements is at least as good as the optimal premium plan for a reinsurance contract with $n$ reinstatements.

Optimality considered here is optimality for the reinsurer. The cedent usually has a different optimality criterion. For example, the cedent might wish to minimize the variance of the total premium; since the variance of the total 
premium is minimal if and only if the total premium is constant, the cedent would in this case prefer a reinsurance contract with no (or free) reinstatements.

Reinsurance contracts with reinstatements can be formulated in various ways. For example, Sundt [1991] considered a reinsurance contract with reinstatements pro rata capita in which, in the notation of the present paper, the total premium is defined as the random variable

$$
\delta:=\pi_{0}\left(1+\frac{1}{h} \sum_{k=1}^{n} c_{k} \min \left\{(S-(k-1) h)^{+}, h\right\}\right)
$$

where $\pi_{0}$ is the initial premium and $c_{1}, \ldots, c_{n} \in[0,1]$ are constants which are fixed in advance and determine the level of the reinstatement premiums with respect to the initial premium; see also Mata [2000]. Sundt studied the question of how to determine the initial premium when the total premium is required to satisfy a certain premium principle like the net premium principle or the standard deviation principle. Letting

$$
\pi_{k}:=\frac{\pi_{0} c_{k}}{h} \min \left\{(S-(k-1) h)^{+}, h\right\}
$$

the total premium can be written as

$$
\delta=\pi_{0}+\sum_{k=1}^{n} \pi_{k} \chi_{\{(k-1) h<S\}}
$$

The model considered by Sundt and the model considered in the present paper are similar in the sense that in both cases it depends on the reinsurer's loss whether or not a certain reinstatement premium is due. The models are nevertheless distinct:

- In the model of Sundt, the reinstatement premium $\pi_{k}$ is due when $S>(k-1) h$ whereas in our model it is due when $S>k h$.

- In the model of Sundt, the reinstatement premiums depend on $S$ and are thus random variables whereas in our model they are constant.

Therefore, our model is simpler than the model of Sundt, but it is this simplification which allows for the selection of an optimal premium plan.

\section{ACKNOWLEDGEMENT}

The authors would like to thank Michael Radtke and Axel Reich for a discussion on reinsurance with reinstatements. They are also grateful to a referee for several suggestions which helped to improve the paper.

\section{REFERENCES}

Hess, K.Th. [2003] Das kollektive Modell der Risikotheorie in der Schadenexzedenten-Rückversicherung. Allg. Statist. Archiv 87, 309-320. 
Hess, K.TH. and Schmidt, K.D. [2001] Credibility-Modelle in Tarifierung und Reservierung. Allg. Statist. Archiv 85, 225-246.

MatA, A.J. [2000] Pricing excess of loss reinsurance with reinstatements. ASTIN Bulletin 30, 349-368.

SchmidT, K.D. [1996] Lectures on Risk Theory. Stuttgart: Teubner.

SchmidT, K.D. [2002] Versicherungsmathematik. Berlin-Heidelberg-New York, Springer.

SundT, B. [1991] On excess of loss reinsurance with reinstatements. Mitt. Verein. Schweiz. Versicherungsmathematiker 91, 51-66.

Klaus Th. Hess and Klaus D. SchmidT

Lehrstuhl für Versicherungsmathemetik

Technische Universität Dresden

D-01062 Dresden

E-mail: schmidt@math.tu-dresden.de 